\title{
Iridium-catalyzed asymmetric hydrogenation of fluorinated olefins using N,P- ligands: A struggle with hydrogenolysis and selectivity
}

\author{
Mattias Engman, Jarle S. Diesen, Alexander Paptchikhine and Pher G. Andersson* \\ Department of Biochemistry and Organic Chemistry, Uppsala University, Box 576, S-751 23 \\ Uppsala.
}

Pher.Andersson@biorg.uu.se

Supporting information

\begin{abstract}
All reactions were run under $\mathrm{N}_{2}$ using flame-dried glassware and magnetic stirring. THF was freshly distilled under $\mathrm{N}_{2}$ from a deepblue solution of sodium-benzophenone ketyl prior to use. $\mathrm{CH}_{2} \mathrm{Cl}_{2}$ was freshly distilled under $\mathrm{N}_{2}$ from powdered $\mathrm{CaH}_{2}$ and diisopropyl ethyl amine was distilled under $\mathrm{N}_{2}$ from $\mathrm{KOH}$ prior to use. Flash chromatography was performed using silica gel $60 \AA$ (37-70 $\left.\mu \mathrm{m}\right)$. Analytical TLC was carried out utilizing $0.25 \mathrm{~mm}$ precoated plates, silica gel $60 \mathrm{UV}_{254}$, and spots were visualized using UV light or ethanolic phosphomolybdic acid (5\%) followed by heating. For NMR spectroscopy, samples were dissolved in $\mathrm{CDCl}_{3}$ and run at room temperature. ${ }^{1} \mathrm{H}(300,400$ or $500 \mathrm{MHz}),{ }^{13} \mathrm{C}(75$ or $100 \mathrm{MHz})$ and ${ }^{19} \mathrm{~F}(282$ or $376 \mathrm{MHz}$ ) NMR spectra were recorded on a 300,400 or $500 \mathrm{MHz}$ spectrometer whereas ${ }^{31} \mathrm{P}(121 \mathrm{MHz}) \mathrm{NMR}$ spectra were recorded on a $300 \mathrm{MHz}$ spectrometer in $\mathrm{C}_{6} \mathrm{D}_{6}$. The relative shifts for phosphorus are reported relative to external $\mathrm{H}_{3} \mathrm{PO}_{4}$. Chemical shifts for protons are reported using the residual $\mathrm{CHCl}_{3}$ as internal reference ( $\delta$ 7.26). Carbon signals are referenced to the shift from the ${ }^{13} \mathrm{C}$ signal of $\mathrm{CDCl} 3(\delta 77.0)$. The chemical shifts for fluorine are reported relative to external $\mathrm{CFCl}_{3}$. Mass spectra were measured at $70 \mathrm{eV}(\mathrm{EI})$. IR spectra was measured using a FT-IR apparatus. Optical rotation was measured using a sodium lamp $(589 \mathrm{~nm})$. Chiral analyses were done by GC-MS $\left(\mathrm{G}-\mathrm{TA}, 90^{\circ} \mathrm{C} 30 \mathrm{~min}, 1^{\circ} \mathrm{C} / \mathrm{min}\right.$ $130^{\circ} \mathrm{C}, 20^{\circ} \mathrm{C} / \mathrm{min} 175^{\circ} \mathrm{C}, 14.5 \mathrm{psi}, 1.5 \mathrm{ml} / \mathrm{min}$ ) or HPLC (Chiralcel OB-H, i.d. $4.6 \mathrm{~mm} \times 25 \mathrm{~cm} 3 \% i$-PrOH: $97 \%$ n-hexane or $5 \% i$ PrOH: $95 \%$ heptane $0.5 \mathrm{~mL} / \mathrm{min}, 220 \mathrm{~nm}$ ). Preparative HPLC was run using YMC, $250 \times 30 \mathrm{~mm}, 10 \mathrm{~mL} / \mathrm{min}, 80 \%$ methanol : $20 \%$ $\mathrm{H}_{2} \mathrm{O}$ or $90 \%$ methanol : $10 \% \mathrm{H}_{2} \mathrm{O}$.
\end{abstract}

Substrate synthesis

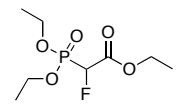

Ethyl 2-(diethoxyphosphoryl)-2-fluoroacetate: ${ }^{1,2}$ Ethyl 2-bromo 2-fluoroacetate (10 g, $\left.52 \mathrm{mmol}\right)$ was added to triethylphosphite (23 $\mathrm{mL}, 131 \mathrm{mmol})$ and heated at $130{ }^{\circ} \mathrm{C}$ for $23 \mathrm{~h}$. The resulting mixture was distilled under low pressure $\left(1.4 \mathrm{mbar}, 75-110{ }^{\circ} \mathrm{C}\right.$ in oil bath for $1 \mathrm{~h}$, heated using a heating gun to maximum temperature $\left.110{ }^{\circ} \mathrm{C}\right)$. The remaining yellowish oil yielded $11.5 \mathrm{~g}(86 \%)$. ${ }^{1} \mathrm{H}$ NMR

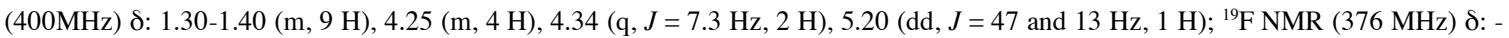


$211(\mathrm{dd}, J=72$ and $47 \mathrm{~Hz}) ;{ }^{13} \mathrm{C}$ NMR $(100 \mathrm{MHz}) \delta: 14.0,16.2(\mathrm{~d}, J=5.9 \mathrm{~Hz}), 62.0,64.0(\mathrm{t}, J=6.4 \mathrm{~Hz}), 84.9(\mathrm{dd}, J=197$ and 159 $\mathrm{Hz}), 164.7$ (dd, $J=22$ and $1.2 \mathrm{~Hz})$.

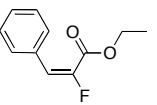

(E)-Ethyl 2-fluoro-3-phenylacrylate: ${ }^{3,4}$ Ethyl 2-(diethoxyphosphoryl)-2-fluoroacetate (10 g, $\left.41 \mathrm{mmol}\right)$ was dissolved in dry THF under $\mathrm{N}_{2}$. The solution was cooled to $0{ }^{\circ} \mathrm{C}$ and $n$-BuLi $(1.6 \mathrm{M}, 25.8 \mathrm{~mL})$ was added. The solution was stirred for $1 \mathrm{~h}$ at $0{ }^{\circ} \mathrm{C}$. Benzaldehyde $(3.5 \mathrm{~mL} 34 \mathrm{mmol})$ was added and the solution was left over night at room temperature. The reaction was quenched with $\mathrm{H}_{2} \mathrm{O}(100 \mathrm{~mL})$, extracted with $\mathrm{CH}_{2} \mathrm{Cl}_{2}(3 \times 100 \mathrm{~mL})$, dried over $\mathrm{MgSO}_{4}$ and evaporated. The product was separated by column chromatography (pentane: $\mathrm{Et}_{2} \mathrm{O}$ 99:1), yielding $1.0 \mathrm{~g}$ of the pure $(E)$-isomer $(13 \%)$ and a 10:1 E:Z mixture $(2.0 \mathrm{~g}) .{ }^{1} \mathrm{H}$ NMR (300

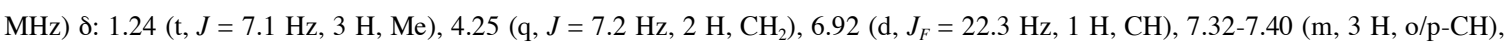
7.43-7.50 (m, $2 \mathrm{H}, \mathrm{m}-\mathrm{CH}) ;{ }^{13} \mathrm{C}$ NMR $(75 \mathrm{MHz}) \delta: 13.8,61.6,121.4\left(\mathrm{~d}, J_{F}=25.6 \mathrm{~Hz}\right), 128.0,128.6,129.6\left(\mathrm{~d}, J_{F}=2.9 \mathrm{~Hz}\right), 131.0\left(\mathrm{~d}, J_{F}\right.$ $=9.4 \mathrm{~Hz}), 147.0(\mathrm{~d}, J=254.7 \mathrm{~Hz}), 160.5(\mathrm{~d}, J=36.4 \mathrm{~Hz}) ;{ }^{19} \mathrm{~F}$ NMR $(282 \mathrm{MHz}) \delta:-117.6(\mathrm{~d}, J=21.3 \mathrm{~Hz})$.

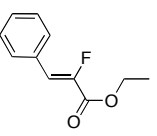

(Z)-Ethyl 2-fluoro-3-phenylacrylate: ${ }^{3,4}$ Ethyl 2-(diethoxyphosphoryl)-2-fluoroacetate (6 g, $24.8 \mathrm{mmol}$ ) was evacuated pump for 15 min, dissolved in dry THF, transferred to a dried round bottomed flask and cooled to $0^{\circ} \mathrm{C} .1 .6 \mathrm{M} n$-BuLi (16.3 mL) was added and the solution was stirred for $1 \mathrm{~h}$ at $0{ }^{\circ} \mathrm{C}$. Benzoyl chloride $(3.0 \mathrm{~mL}, 26.0 \mathrm{mmol})$ was added and the mixture was stirred at RT for $4 \mathrm{~h}$. The reaction was quenched with $\mathrm{H}_{2} \mathrm{O}(100 \mathrm{~mL})$, extracted with $\mathrm{CH}_{2} \mathrm{Cl}_{2}(3 \times 75 \mathrm{~mL})$, dried over $\mathrm{MgSO}_{4}$ and evaporated giving an oil. (Sometimes the resulting oil needed to be separated by column chromatography in $\mathrm{Et}_{2} \mathrm{O}$ :pentane 40:60 with a yield of $76 \%$ ). The crude oil was dissolved in ethanol $(180 \mathrm{~mL})$ and cooled to $-78^{\circ} \mathrm{C}$. Sodium borohydride $(960 \mathrm{mg}, 37.8 \mathrm{mmol})$ was added in ethanol (20 $\mathrm{mL}$ ) and the solution was stirred for $1 \mathrm{~h}$ at $-78{ }^{\circ} \mathrm{C}$, then for $4 \mathrm{~h}$ at RT. It was then quenched with $\mathrm{NH}_{4} \mathrm{Cl}$ (aq, satd., $200 \mathrm{~mL}$ ), extracted with $\mathrm{CH}_{2} \mathrm{Cl}_{2}$, dried over $\mathrm{MgSO}_{4}$, evaporated, redissolved in $\mathrm{CH}_{2} \mathrm{Cl}_{2}$, washed with water (to remove inorganic salts), dried over $\mathrm{MgSO}_{4}$ and evaporated. The crude oil was separated by column chromatography (pentane: $\mathrm{Et}_{2} \mathrm{O}$ 95:5) yielding the pure $Z$ isomer (2.1 $\left.\mathrm{g}, 44 \%\right)$. ${ }^{1} \mathrm{H}$ NMR (300 MHz) $\delta: 1.39$ (t, $\left.J=7.2 \mathrm{~Hz}, 3 \mathrm{H}, \mathrm{Me}\right), 4.36$ (q, $\left.J=7.1 \mathrm{~Hz}, 2 \mathrm{H}, \mathrm{CH}_{2}\right), 6.93\left(\mathrm{~d}, J_{F}=35.2 \mathrm{~Hz}, 1 \mathrm{H}, \mathrm{CH}\right), 7.36-7.46(m, 3$ $\mathrm{H}, o / p-\mathrm{CH}), 7.62-7.68(m, 2 \mathrm{H}, m-\mathrm{CH}) ;{ }^{13} \mathrm{C} \mathrm{NMR}(75 \mathrm{MHz}) \delta: 14.2,61.8,117.4\left(\mathrm{~d}, J_{F}=4.8 \mathrm{~Hz}\right), 128.8,129.6\left(\mathrm{~d}, J_{F}=2.8 \mathrm{~Hz}\right), 130.3$ $\left(\mathrm{d}, J_{F}=8.4 \mathrm{~Hz}\right), 131.1\left(\mathrm{~d}, J_{F}=4.3 \mathrm{~Hz}\right), 147.0\left(\mathrm{~d}, J_{F}=267.6 \mathrm{~Hz}\right), 161.4\left(\mathrm{~d}, J_{F}=34.4 \mathrm{~Hz}\right) ;{ }^{19} \mathrm{~F} \mathrm{NMR}(282 \mathrm{MHz}) \delta:-125.7(\mathrm{~d}, J=35.1$ $\mathrm{Hz})$.

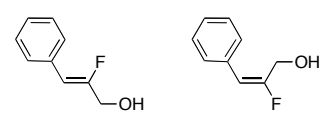

(E) or (Z) 2-Fluoro-3-phenylprop-2-en-1-ol: ${ }^{5}(E)$-Ethyl 2-fluoro-3-phenylacrylate $(1.1 \mathrm{~g}, 5.7 \mathrm{mmol})$ was dissolved in dry THF (40 $\mathrm{mL})$ in dry glassware. DIBAL $(1.5 \mathrm{M}$ in toluene, $11.3 \mathrm{~mL})$ was added at $0{ }^{\circ} \mathrm{C}$. The solution was stirred at RT over night, quenched with Rochelles salt under vigorous stirring, extracted with $\mathrm{CH}_{2} \mathrm{Cl}_{2}(3 \times 50 \mathrm{~mL})$, washed with water $(2 \times 50 \mathrm{~mL})$, dried over $\mathrm{MgSO}_{4}$ and evaporated. Product was separated by column chromatography (pentane: $\mathrm{Et}_{2} \mathrm{O}$ 90:10 to 50:50). Yield: $725 \mathrm{mg}(84 \%)$. (Z) isomer: ${ }^{1} \mathrm{H}$ NMR (300 MHz) 8: $2.74(\mathrm{br} \mathrm{s}, 1 \mathrm{H}, \mathrm{OH}), 4.26\left(\mathrm{~d}, J=14.3,2 \mathrm{H}, \mathrm{CH}_{2}\right), 5.78(\mathrm{~d}, J=38.8 \mathrm{~Hz}, 1 \mathrm{H}, \mathrm{CH}), 7.21-7.40$ (m, $\left.3 \mathrm{H}, \mathrm{Ar}\right)$, 7.48-7.56 (m, $2 \mathrm{H}, \mathrm{Ar}) ;{ }^{13} \mathrm{C}$ NMR (75 MHz) $\delta: 53.4,61.6(\mathrm{~d}, J=32.7 \mathrm{~Hz}), 107.3(\mathrm{~d}, J=6.6 \mathrm{~Hz}), 127.4(\mathrm{~d}, J=2.3 \mathrm{~Hz}), 128.4(\mathrm{~d}, J=$ 
$7.3 \mathrm{~Hz}), 132.7(\mathrm{~d}, J=3.0 \mathrm{~Hz}), 158.2(\mathrm{~d}, J=266.9 \mathrm{~Hz}) ;{ }^{19} \mathrm{~F}$ NMR $(282 \mathrm{MHz}) \delta:-113.5(\mathrm{dt}, J=38.9$ and $14.2 \mathrm{~Hz})$; $(E)$ isomer: ${ }^{1} \mathrm{H}$ NMR (300 MHz) 8: 2.27 (br s, $1 \mathrm{H}, \mathrm{OH}), 4.37\left(\mathrm{~d}, J=22.1 \mathrm{~Hz}, 2 \mathrm{H}, \mathrm{CH}_{2}\right), 6.41(\mathrm{~d}, J=19.8 \mathrm{~Hz}, 1 \mathrm{H}, \mathrm{CH}), 7.21-7.40(m, 5 \mathrm{H}, \mathrm{Ar}) ;{ }^{13} \mathrm{C} \mathrm{NMR}$ $(75 \mathrm{MHz}) \delta: 58.2(\mathrm{~d}, J=29.3 \mathrm{~Hz}), 111.4(\mathrm{~d}, J=26.0 \mathrm{~Hz}), 127.4(\mathrm{~d}, J=0.8 \mathrm{~Hz}), 128.5,128.6,132.9(\mathrm{~d}, J=12.6 \mathrm{~Hz}), 159.5(\mathrm{~d}, J=$ $253.7 \mathrm{~Hz}) ;{ }^{19} \mathrm{~F}$ NMR $(282 \mathrm{MHz}) \delta:-109.4(\mathrm{q}, J=21.3 \mathrm{~Hz})$

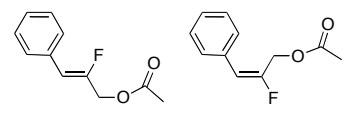

(E) or (Z)-2-Fluoro-3-phenylbut-2-enyl acetate: ${ }^{6,7,8}(Z)$ 2-Fluoro-3-phenylprop-2-en-1-ol (800 mg, $5.3 \mathrm{mmol}$ ) was dissolved in 20 $\mathrm{mL}$ pyridine at $0{ }^{\circ} \mathrm{C}$. Acetic anhydride $(1.4 \mathrm{~mL}, 16 \mathrm{mmol})$ was added slowly at $0{ }^{\circ} \mathrm{C}$. The solution was stirred at room temperature for $24 \mathrm{~h}$ and quenched with $\mathrm{NaHCO}_{3}$ solution (aq, sat'd., $\left.40 \mathrm{~mL}\right)$. It was then diluted with water $(20 \mathrm{~mL})$, extracted with $\mathrm{CH}_{2} \mathrm{Cl}_{2}(3 \times 50$ $\mathrm{mL})$, dried $\left(\mathrm{MgSO}_{4}\right)$, evaporated and co-evaporated with toluene $(2 \times 10 \mathrm{~mL})$ to remove pyridine. Product was separated by column chromatography (pentane: $\mathrm{Et}_{2} \mathrm{O} 90: 10$ ) yielding $0.49 \mathrm{~g} \mathrm{(48 \% )}$ of pure (Z)-isomer. (Z)-isomer: ${ }^{1} \mathrm{H}$ NMR (300 MHz) $\delta: 2.14$ (s, $3 \mathrm{H}$, $\mathrm{Me}), 4.74\left(\mathrm{~d}, J=17.8 \mathrm{~Hz}, 2 \mathrm{H}, \mathrm{CH}_{2}\right), 5.85(\mathrm{~d}, J=37.3 \mathrm{~Hz}, 1 \mathrm{H}, \mathrm{CH}), 7.24-7.40(m, 3 \mathrm{H}, o / p-\mathrm{CH}), 7.49-7.56(m, 2 \mathrm{H}, m-\mathrm{CH}) ;{ }^{13} \mathrm{C}$ NMR (75 MHz) 8: 20.7, 62.9 (d, $J=31.9 \mathrm{~Hz}), 111.0(\mathrm{~d}, J=6.7 \mathrm{~Hz}), 127.9(\mathrm{~d}, J=2.6 \mathrm{~Hz}), 128.5,128.9(\mathrm{~d}, J=7.6 \mathrm{~Hz}), 132.2(\mathrm{~d}, J=$ $3.2 \mathrm{~Hz}), 154.0(\mathrm{~d}, J=264.9 \mathrm{~Hz}), 170.4 ;{ }^{19} \mathrm{~F}$ NMR $(282 \mathrm{MHz}) \delta:-111.6(\mathrm{dt}, J=37.2$ and $17.6 \mathrm{~Hz})$; $(E)$-isomer: ${ }^{1} \mathrm{H}$ NMR $(300 \mathrm{MHz}) \delta$ : $2.14(\mathrm{~s}, 3 \mathrm{H}, \mathrm{Me}), 4.82\left(\mathrm{~d}, J_{F}=21.6 \mathrm{~Hz}, 2 \mathrm{H}, \mathrm{CH}_{2}\right), 6.53\left(\mathrm{~d}, J_{F}=13.9 \mathrm{~Hz}, 1 \mathrm{H}, \mathrm{CH}\right), 7.19-7.25(m, 2 \mathrm{H}, \mathrm{Ar}), 7.28-7.41(m, 3 \mathrm{H}, \mathrm{Ar})$; ${ }^{13} \mathrm{C}$ NMR (75 MHz) 8: 20.7, $59.4\left(\mathrm{~d}, J_{F}=59.6 \mathrm{~Hz}\right), 114.0(\mathrm{~d}, J=25.5 \mathrm{~Hz}), 127.7,128.5(\mathrm{~d}, J=2.8 \mathrm{~Hz}), 128.7,132.4(\mathrm{~d}, J=11.6 \mathrm{~Hz})$, $155.5(\mathrm{~d}, J=254.2 \mathrm{~Hz}), 170.5 ;{ }^{19} \mathrm{~F}$ NMR $(282 \mathrm{MHz}) \delta:-107.4(\mathrm{dd}, J=41.5$ and $21.4 \mathrm{~Hz})$

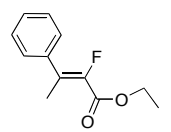

(E) and $(\boldsymbol{Z})$-Ethyl 2-fluoro-3-phenylbut-2-enoate:9,10 Ethyl 2-(diethoxyphosphoryl)-2-fluoroacetate $(1.0 \mathrm{~g}, 4.1 \mathrm{mmol})$ was dissolved in dry THF under $\mathrm{N}_{2}$. The solution was cooled to $0{ }^{\circ} \mathrm{C}$ and $n$-BuLi $(1.6 \mathrm{M}, 2.6 \mathrm{~mL})$ was added. The solution was left for $1 \mathrm{~h}$ at $0{ }^{\circ} \mathrm{C}$. Acetophenone $(0.4 \mathrm{~mL}, 3.4 \mathrm{mmol})$ was added and the solution was left over night at RT. The reaction was quenched with $\mathrm{H}_{2} \mathrm{O}(50$ $\mathrm{mL})$, extracted with $\mathrm{CH}_{2} \mathrm{Cl}_{2}(3 \times 75 \mathrm{~mL})$, dried over $\mathrm{MgSO}_{4}$ and the solvent was evaporated in vacuo. Product was separated by column chromatography (pentane: $\mathrm{Et}_{2} \mathrm{O} 100: 0$ to $\left.95: 5\right)$ yielding $0.55 \mathrm{~g}(64 \%)$ of the pure $(E)$ isomer and $0.12 \mathrm{~g}(14 \%)$ of pure $(Z)$ isomer, both products were isolated as colorless oils. (Z)-isomer: ${ }^{1} \mathrm{H}$ NMR $(400 \mathrm{MHz}) \delta: 1.38(\mathrm{t}, J=7.3 \mathrm{~Hz}, 3 \mathrm{H}, \mathrm{Me}), 2.45$ (d, $J=3.5$ $\mathrm{Hz}, 3 \mathrm{H}, \mathrm{MeC}), 4.34$ (q, J = 7.2 Hz, $\left.2 \mathrm{H}, \mathrm{CH}_{2}\right), 7.30-7.41$ (m, $\left.5 \mathrm{H}, \mathrm{Ar}\right) ;{ }^{13} \mathrm{C} \mathrm{NMR}(100 \mathrm{MHz}) \delta: 14.2,18.3,61.3,127.9(\mathrm{~d}, J=4.0 \mathrm{~Hz})$, 128.2, 128.3, $130.6(\mathrm{~d}, J=11.4 \mathrm{~Hz}), 137.6,144.6,161.8(\mathrm{~d}, J=34.3 \mathrm{~Hz}) ;{ }^{19} \mathrm{~F}$ NMR $(376 \mathrm{MHz}) \delta:-126.2(\mathrm{q}, J=3.8 \mathrm{~Hz}) ;(E)$ isomer:

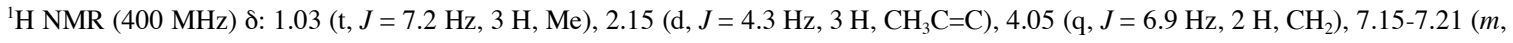
$2 \mathrm{H}, \mathrm{Ar}), 7.29-7.39(m, 3 \mathrm{H}, \mathrm{Ar}) ;{ }^{13} \mathrm{C}$ NMR $(100 \mathrm{MHz}) \delta: 13.6,19.3$ (d, $\left.J=6.5 \mathrm{~Hz}\right), 61.0,127.4(\mathrm{~d}, J=3.1 \mathrm{~Hz}), 127.7,128.0,131.4(\mathrm{~d}$, $J=17.0 \mathrm{~Hz}), 138.5(\mathrm{~d}, J=5.5 \mathrm{~Hz}), 144.2(\mathrm{~d}, J=251.3 \mathrm{~Hz}), 160.6(\mathrm{~d}, J=36.0 \mathrm{~Hz}) ;{ }^{19} \mathrm{~F}$ NMR $(376 \mathrm{MHz}) \delta:-124.6(\mathrm{q}, J=4.8 \mathrm{~Hz})$. The $\mathrm{E} / \mathrm{Z}$ conformations of the olefins were confirmed by ${ }^{1} \mathrm{H}^{-19} \mathrm{~F}$ NOE experiments. 


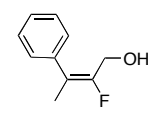

(E)-2-Fluoro-3-phenylbut-2-en-1-ol: ${ }^{11}(E)$-Ethyl 2-fluoro-3-phenylbut-2-enoate $(0.20 \mathrm{~g}, 1.0 \mathrm{mmol})$ was dissolved in dry THF (5 mL) in dry glassware. DIBAL $(1.5 \mathrm{M}$ in toluene, $2.1 \mathrm{~mL})$ was added at $0{ }^{\circ} \mathrm{C}$ and stirred at RT over night. It was then quenched with Rochelles salt under vigorous stirring, extracted with $\mathrm{CH}_{2} \mathrm{Cl}_{2}(3 \times 50 \mathrm{~mL})$, washed with water $(2 \times 50 \mathrm{~mL})$, dried over $\mathrm{MgSO}$ and evaporated. Product was separated by column chromatography (pentane/ $\mathrm{Et}_{2} \mathrm{O} 90: 10$ ), yielding $0.10 \mathrm{~g}(64 \%)$ of a colorless oil. ${ }^{1} \mathrm{H}$

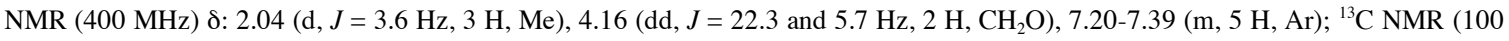
MHz) $\delta: 16.1(\mathrm{~d}, J=6.8 \mathrm{~Hz}), 58.8(\mathrm{~d}, J=30.2 \mathrm{~Hz}), 118.9(\mathrm{~d}, J=18.3 \mathrm{~Hz}), 127.4,128.2(\mathrm{~d}, J=3.0 \mathrm{~Hz}), 128.4,138.8(\mathrm{~d}, J=8.3 \mathrm{~Hz})$, $154.8(\mathrm{~d}, J=250.6 \mathrm{~Hz}) ;{ }^{19} \mathrm{~F}$ NMR $(376 \mathrm{MHz}) \delta:-118.0(\mathrm{tq}, J=22.4$ and $3.8 \mathrm{~Hz})$

\section{Hydrogenations}

\section{General procedure for racemate synthesis}

A round-bottomed flask was charged with fluoro olefin $(100 \mathrm{mg}), \mathrm{Pd} / \mathrm{C}(2 \mathrm{~mol} \%)$ and EtOH (abs, $2 \mathrm{~mL})$ or EtOAc. The mixture was hydrogenated with $\mathrm{H}_{2}(1 \mathrm{~atm})$ for $12-72 \mathrm{~h}$. The product mixture was filtered through a plug of Celite ${ }^{\circledR}$ and the solvent was removed in vacuo.

\section{General procedure for hydrogenations}

A vial was charged with substrate $(0.1-0.5 \mathrm{mmol})$ and catalyst $(0.5-2 \mathrm{~mol} \%)$ and $\mathrm{CH}_{2} \mathrm{Cl}_{2}(2 \mathrm{~mL})$ was added. For 100 bar hydrogenations: The vial was placed in high-pressure steel equipment, which was purged three times with $\mathrm{H}_{2}$ before it was pressurized to 100 bars, heated to $40{ }^{\circ} \mathrm{C}$ and held at this pressure for $12-72$ hours. One hour before the end of the reaction, the heating was turned off. The pressure was released and the solvent was evaporated off. For hydrogenations at pressures up to 30 bar: The vial was placed in a Endeavor ${ }^{\mathrm{TM}}$ Catalyst screening system. Vessels were purged three times with $\operatorname{Ar}(10 \mathrm{~atm})$, and then flushed and pressurized with $\mathrm{H}_{2}$ to working pressure and stirred at $700 \mathrm{rpm}$ for $12-24$ hours. In all cases, conversions were measured by ${ }^{1} \mathrm{H}$ NMR after evaporation of solvent. $1.5 \mathrm{~mL}$ of $\mathrm{Et}_{2} \mathrm{O}$ :pentane (1:1) was added, the solution was filtered through a short plug of silica. The silica plug was rinsed with $3 \mathrm{~mL} \mathrm{Et} t_{2} \mathrm{O}$ :pentane (1:1). The solvent was evaporated and the ee was determined by GC-MS (G-TA, $90^{\circ} \mathrm{C} 30 \mathrm{~min}$, $1{ }^{\circ} \mathrm{C} / \mathrm{min} 130^{\circ} \mathrm{C}, 20^{\circ} \mathrm{C} / \mathrm{min} 175^{\circ} \mathrm{C}, 14.5 \mathrm{psi}, 1.5 \mathrm{ml} / \mathrm{min}$ ) or HPLC (Chiralcel OB-H, i.d. $4.6 \mathrm{~mm} \times 25 \mathrm{~cm} 3 \%$ i-PrOH: $97 \% n$-hexane or $5 \%$-PrOH: $95 \%$ heptane $0.5 \mathrm{~mL} / \mathrm{min}, 220 \mathrm{~nm}$ ). Preparative HPLC was run using YMC, $250 \times 30 \mathrm{~mm}, 10 \mathrm{~mL} / \mathrm{min}, 80 \%$ methanol: $20 \% \mathrm{H}_{2} \mathrm{O}$ or $90 \%$ methanol: $10 \% \mathrm{H}_{2} \mathrm{O}$. The absolute configurations of entries 1-3 were derived from the optical rotation of entry 3 and the elution order in HPLC and GC respectively.

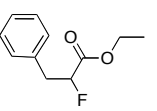

Hydrogenations of racemic ethyl 2-fluoro-3-phenylpropanoate: To ethyl 2-fluoro-3-phenylpropanoate (20 mg, $0.1 \mathrm{mmol})$ was added oxazole complex $\mathbf{V}(3.9 \mathrm{mg}, 2.5 \mu \mathrm{mol}, 2,5 \mathrm{~mol} \%)$. This mixture was dissolved in trifluorotoluene $(2 \mathrm{~mL})$ and hydrogenated at 100 bar, $40{ }^{\circ} \mathrm{C}$ for $52 \mathrm{~h}$. Crude ${ }^{1} \mathrm{H}-\mathrm{NMR}$ showed no trace of defluorinated product. 


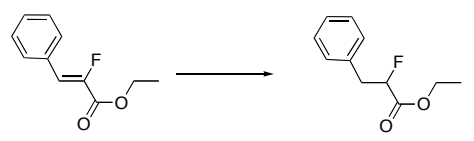

Hydrogenation of (Z)-ethyl 2-fluoro-3-phenylacrylate (Table 1, entry 1):

Complex I: Conditions: 100 bar, $40{ }^{\circ} \mathrm{C}, 72 \mathrm{~h}, 1 \mathrm{~mol} \%$ of catalyst, $\mathrm{CH}_{2} \mathrm{Cl}_{2} ;(R)-2$-fluoro-3-phenylpropanoate. ${ }^{12}$ The product was treated with DIBAL ( 3 eq DIBAL, $20 \mathrm{mg}$ scale, $0.5 \mathrm{~mL}$ THF) to generate the alcohol and purified by prep. HPLC ( $80 \%$ methanol : 20 $\% \mathrm{H}_{2} \mathrm{O}$ ). Absolute configuration and ee were determined by chiral HPLC analysis ( $3 \% i$-PrOH: $97 \% n$-hexane). $\mathrm{t}_{\mathrm{R}}=36.6 \mathrm{~min}$ (major), $38.7 \mathrm{~min}$ (minor). The $e e$ was confirmed on GC-MS: $\mathrm{t}_{\mathrm{R}}=34.2 \mathrm{~min}$ (major), $38.5 \mathrm{~min}$ (minor)

Complex IV: Conditions: 100 bar, RT, 66 h, $0.5 \mathrm{~mol} \%, \mathrm{CH}_{2} \mathrm{Cl}_{2}$; No conversion ( $\left.{ }^{1} \mathrm{H}-\mathrm{NMR}\right)$.

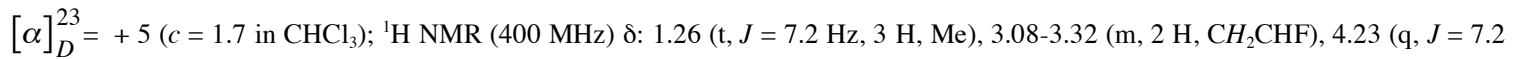
$\mathrm{Hz}, 2 \mathrm{H}, \mathrm{CH}_{2} \mathrm{Me}$ ), 5.09 (ddd, $J=48.7,7.6$ and $\left.4.1 \mathrm{~Hz}, 1 \mathrm{H}, \mathrm{CHF}\right), 7.23-7.36$ (m, $\left.5 \mathrm{H}, \mathrm{Ar}\right) ;{ }^{13} \mathrm{C}$ NMR $(100 \mathrm{MHz}) \delta: 14.1,38.7$ (d, $J=$ $21.2 \mathrm{~Hz}), 61.5,89.2$ (d, $J=187.4 \mathrm{~Hz}), 127.1,128.5,129.4,135.1,169.2(\mathrm{~d}, J=23.6 \mathrm{~Hz}) ;{ }^{19} \mathrm{~F}$ NMR $(282 \mathrm{MHz}) \delta:-190.2$ (ddd, $J=$ $48.8,28.6$ and $24.3 \mathrm{~Hz}$ ).

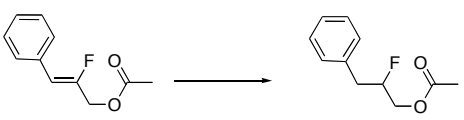

Hydrogenation of (Z)- 2-fluoro-3-phenylbut-2-enyl acetate (Table 1, entry 2):

Complex I: Conditions: 30 bar, RT, $12 \mathrm{~h}, 1 \mathrm{~mol} \%, \mathrm{CH}_{2} \mathrm{Cl}_{2} ;(R)$-2-fluoro-3-phenylpropyl acetate. The product was refluxed in EtOH with $\mathrm{K}_{2} \mathrm{CO}_{3}$ for $30 \mathrm{~min}$ to generate the alcohol and purified by prep. HPLC $\left(80 \%\right.$ methanol : $\left.20 \% \mathrm{H}_{2} \mathrm{O}\right)$ to determine the absolute configuration on chiral HPLC ( $3 \% i$-PrOH: $97 \% n$-hexane), $\mathrm{t}_{\mathrm{R}}=36.1 \mathrm{~min}$ (major), $38.2 \mathrm{~min}$ (minor). The $e e$ was determined by GCMS, $\mathrm{t}_{\mathrm{R}}=39.2 \min$ (major), $40.1 \mathrm{~min}$ (minor).

Complex VI: Conditions: 30 bar, room temperature, $24 \mathrm{~h}, 1$ mol- $\%, \mathrm{CH}_{2} \mathrm{Cl}_{2} ;(R)$-2-fluoro-3-phenylpropyl acetate. The ee was determined by GC-MS, $\mathrm{t}_{\mathrm{R}}=39.3 \mathrm{~min}$ (major), $40.1 \mathrm{~min}$ (minor).

$[\alpha]_{D}^{24}=-4\left(c=1.0\right.$ in $\mathrm{CHCl}_{3}, 74 \%$ ee); $\mathrm{R}_{\mathrm{f}}=0.42\left(\mathrm{Et}_{2} \mathrm{O} / \mathrm{Pentane} 10: 90\right) ; \mathrm{IR}$ (neat) $v_{\max } 3031,2953,1742,1497,1455,1369,1233$, 1084, 1048, 748 and $701 \mathrm{~cm}^{-1}$; ${ }^{1} \mathrm{H}$ NMR (400 MHz) $\delta: 2.11$ (s, $3 \mathrm{H}, \mathrm{Me}$ ), 2.95 (ddd, $J=24.0,14.3$ and $5.7 \mathrm{~Hz}, 1 \mathrm{H}, \mathrm{CH}_{2} \mathrm{O}$ ), 3.05 (ddd, $J=18.3,14.1$ and $\left.7.1 \mathrm{~Hz}, 1 \mathrm{H}, \mathrm{CH}_{2} \mathrm{O}\right), 4.14\left(\mathrm{ddd}, J=21.6,12.5\right.$ and $\left.6.5 \mathrm{~Hz}, 1 \mathrm{H}, \mathrm{CH}_{2} \mathrm{CHF}\right), 4.26(\mathrm{ddd}, J=25.9,12.5 \mathrm{and} 2.9 \mathrm{~Hz}, 1 \mathrm{H}$, $\left.\mathrm{CH}_{2} \mathrm{CHF}\right), 4.89$ (m, $\left.1 \mathrm{H}, \mathrm{CHF}\right), 7.14-7.37$ (m, $\left.5 \mathrm{H}, \mathrm{Ar}\right) ;{ }^{13} \mathrm{C} \mathrm{NMR}(100 \mathrm{MHz}) \delta: 20.8,37.9$ (d, $\left.J=21.2 \mathrm{~Hz}\right), 65.0(\mathrm{~d}, J=23.0 \mathrm{~Hz}), 91.3$ $(\mathrm{d}, J=176.6 \mathrm{~Hz}), 126.9,128.6,129.2,135.7$ (d, $J=5.4 \mathrm{~Hz}), 170.7 ;{ }^{19} \mathrm{~F}$ NMR $(282 \mathrm{MHz}) \delta:-185.5(\mathrm{~m})$; MS (EI) $(m / z)($ rel. intensity) $196\left(\mathrm{M}^{+}+1,14 \%\right), 176(63 \%), 133(100 \%), 116(74 \%)$. 


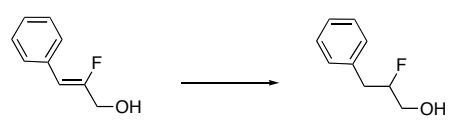

Hydrogenation of (Z)-2-fluoro-3-phenylprop-2-en-1-ol (Table 1, entry 3):

Complex I: Conditions: 20 bar, RT, 24 h, 0.5 mol \%, $\mathrm{CH}_{2} \mathrm{Cl}_{2} ;(R)$-2-fluoro-3-phenylpropan-1-ol. ${ }^{13}$ The $e e$ was determined on chiral HPLC ( $3 \%$-PrOH: $97 \% \mathrm{n}$-hexane), $\mathrm{t}_{\mathrm{R}}=36.0 \mathrm{~min}$ (major), $39.5 \mathrm{~min}$ (minor). The absolute configuration was determined from optical rotation.

Complex VI: Conditions: 20 bar, room temperature, $18 \mathrm{~h}, 0.5 \mathrm{~mol}-\%, \mathrm{CH}_{2} \mathrm{Cl}_{2} ;(R)-2$-fluoro-3-phenylpropyl acetate. The ee and absolute configuration were determined by $\operatorname{HPLC}\left(3 \% i\right.$-PrOH: $97 \% \mathrm{n}$-hexane), $\mathrm{t}_{\mathrm{R}}=36.0 \min$ (major), $39.5 \min$ (minor) and the ee was confirmed on GC-MS, $\mathrm{t}_{\mathrm{R}}=31.2 \mathrm{~min}$ (minor), $31.5 \mathrm{~min}$ (major).

$[\alpha]_{D}^{24}=+12\left(c=1.0\right.$ in $\mathrm{CHCl}_{3}, 80 \%$ ee $) ;{ }^{1} \mathrm{H}$ NMR $(400 \mathrm{MHz}) \delta: 1.96(\mathrm{br} \mathrm{s}, 1 \mathrm{H}, \mathrm{OH}), 2.87-3.10\left(\mathrm{~m}, 2 \mathrm{H}, \mathrm{CH}_{2} \mathrm{CHF}\right), 3.60-3.85(\mathrm{~m}, 2$ $\left.\mathrm{H}, \mathrm{CH}_{2} \mathrm{O}\right), 4.78$ (ddq, $J=48.7,2.9$ and $\left.6.0 \mathrm{~Hz}, 1 \mathrm{H}, \mathrm{CHF}\right), 7.16-7.39(\mathrm{~m}, 5 \mathrm{H}, \mathrm{Ar}) ;{ }^{13} \mathrm{C} \mathrm{NMR}(100 \mathrm{MHz}) \delta: 37.4,64.1(\mathrm{~d}, J=21.9 \mathrm{~Hz})$, $94.6(\mathrm{~d}, J=171.2 \mathrm{~Hz}), 126.8,128.6,129.3,136.3(\mathrm{~d}, J=5.9 \mathrm{~Hz}) ;{ }^{19} \mathrm{~F}$ NMR $(282 \mathrm{MHz}) \delta:-188.0(\mathrm{~m})$.

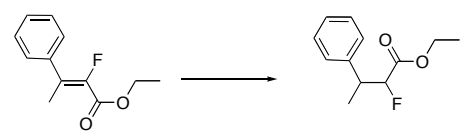

Hydrogenation of (Z)-ethyl 2-fluoro-3-phenylbut-2-enoate (Table 1, entry 4):

Complex I: Conditions: 100 bar, $40^{\circ} \mathrm{C}, 72 \mathrm{~h}, 0.5$ mol- $\%, \mathrm{CH}_{2} \mathrm{Cl}_{2}$; (-)-(2R,3S)-threo-ethyl 2-fluoro-3-phenylbutanoate. ${ }^{14}$ The ee was determined by GC-MS, $\mathrm{t}_{\mathrm{R}}=37.7 \mathrm{~min}$ (major), $42.6 \mathrm{~min}$ (minor).

Complex VI: Conditions: 100 bar, $40^{\circ} \mathrm{C}, 72 \mathrm{~h}, 2$ mol- $\%, \mathrm{CH}_{2} \mathrm{Cl}_{2}$; threo-ethyl 2-fluoro-3-phenylbutanoate. The ee was determined by GC-MS, $\mathrm{t}_{\mathrm{R}}=37.7 \mathrm{~min}, 42.6 \mathrm{~min}$ (racemic)

$[\alpha]_{D}^{24}=-1\left(c=1.0\right.$ in $\mathrm{CHCl}_{3}, 57 \%$ ee $) ;{ }^{1} \mathrm{H}$ NMR $(500 \mathrm{MHz}) \delta: 1.21\left(\mathrm{t}, J=7.2 \mathrm{~Hz}, 3 \mathrm{H}, \mathrm{CH}_{3}\right), 1.38\left(\mathrm{~d}, J=7.2 \mathrm{~Hz}, 3 \mathrm{H}, \mathrm{CH}_{3}\right), 3.35$ (ddq, $J=26.9,7.2$ and $4.3 \mathrm{~Hz}, 1 \mathrm{H}$ ), 4.19 (dq, $J=7.2$ and $2.8 \mathrm{~Hz}, 2 \mathrm{H}, \mathrm{CH}_{2}$ ), 4.95 (dd, $J=49.0$ and $4.3 \mathrm{~Hz}, 1 \mathrm{H}, \mathrm{CHF}$ ), 7.24-7.40 (m, $5 \mathrm{H}, \mathrm{Ar}-\mathrm{H}) ;{ }^{13} \mathrm{C}$ NMR $(75 \mathrm{MHz}) \delta: 14.0,14.9$ (d, $\left.J=5.5 \mathrm{~Hz}\right), 42.3$ (d, $\left.J=20.6 \mathrm{~Hz}\right), 61.4,92.5(\mathrm{~d}, J=190 \mathrm{~Hz}), 127.2,127.8(\mathrm{~d}, J=$ $1.2), 128.5,141.1(\mathrm{~d}, J=2.3), 169.1(\mathrm{~d}, J=23.9 \mathrm{~Hz}) ;{ }^{19} \mathrm{~F} \mathrm{NMR}(282 \mathrm{MHz}) \delta:-198.2(\mathrm{dd}, J=26.5 \mathrm{and} 48.8 \mathrm{~Hz})$.

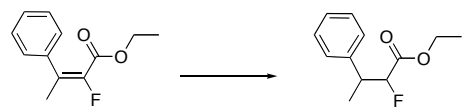

Hydrogenation of (E)-ethyl 2-fluoro-3-phenylbut-2-enoate (Table 1, entry 5): 
Complex I: Conditions: 100 bar, $40{ }^{\circ} \mathrm{C}, 72 \mathrm{~h}, 0.5 \mathrm{~mol} \%, \mathrm{CH}_{2} \mathrm{Cl}_{2}$; (+)-(2S,3S)-erythro-ethyl 2-fluoro-3-phenylbutanoate. ${ }^{14}$ The $e e$ was determined by GC-MS, $\mathrm{t}_{\mathrm{R}}=39.0 \mathrm{~min}$ (major), $42.6 \mathrm{~min}$ (minor).

Complex VI: Conditions: 100 bar, $40{ }^{\circ} \mathrm{C}, 72 \mathrm{~h}, 2 \mathrm{~mol} \%, \mathrm{CH}_{2} \mathrm{Cl}_{2}$; erythro-ethyl 2-fluoro-3-phenylbutanoate. The $e e$ was determined by GC-MS, $\mathrm{t}_{\mathrm{R}}=39.0 \mathrm{~min}, 42.6 \mathrm{~min}$ (racemic)

$[\alpha]_{D}^{24}=+27\left(c=0.7\right.$ in $\left.\mathrm{CHCl}_{3}, 74 \% e e\right) ;{ }^{1} \mathrm{H}$ NMR $(500 \mathrm{MHz}) \delta: 1.14(\mathrm{t}, J=7.2,3 \mathrm{H}, \mathrm{Me}), 1.45\left(\mathrm{~d}, J=7.3 \mathrm{~Hz}, 3 \mathrm{H}, \mathrm{CH}_{3}\right), 3.36(\mathrm{ddq}$, $J=4.6,7.3$ and $28.5 \mathrm{~Hz}, 1 \mathrm{H}), 4.11\left(\mathrm{dq}, J=3.3\right.$ and $\left.7.1 \mathrm{~Hz}, 2 \mathrm{H}, \mathrm{CH}_{2}\right), 5.00(\mathrm{dd}, J=4.6$ and $48.9 \mathrm{~Hz}), 7.15-7.75(\mathrm{~m}, 5 \mathrm{H}, \mathrm{Ar}) ;{ }^{13} \mathrm{C}$

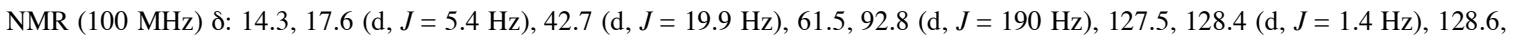
140.2, $169.1(\mathrm{~d}, J=23.7 \mathrm{~Hz}) ;{ }^{19} \mathrm{~F}$ NMR $(376 \mathrm{~Hz}) \delta:-197.9(\mathrm{dd}, J=28.8$ and $48.8 \mathrm{~Hz})$.

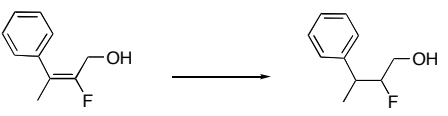

Hydrogenation of (E)-2-fluoro-3-phenylbut-2-en-1-ol (Table 1, entry 6):

Complex I: Conditions: 100 bar, $40{ }^{\circ} \mathrm{C}, 72 \mathrm{~h}, 1 \mathrm{~mol} \%, \mathrm{CH}_{2} \mathrm{Cl}_{2}$; (+)-(2S,3S)-erythro-2-fluoro-3-phenylbutan-1-ol. The ee was determined by chiral HPLC (5 \% $i$-PrOH: $95 \%$ heptane), $\mathrm{t}_{\mathrm{R}}=21.9 \mathrm{~min}$ (minor), $22.8 \mathrm{~min}$ (major). The absolute configuration was derived from comparison with literature data. ${ }^{15}$

Complex VI: Conditions: 100 bar, $40{ }^{\circ} \mathrm{C}, 72 \mathrm{~h}, 1 \mathrm{~mol} \%, \mathrm{CH}_{2} \mathrm{Cl}_{2}$; (+)-(2S,3S)-erythro-2-fluoro-3-phenylbutan-1-ol. The ee was determined by chiral HPLC (5\% $i$-PrOH: $95 \%$ heptane), $\mathrm{t}_{\mathrm{R}}=21.9 \mathrm{~min}$ (minor), $22.8 \mathrm{~min}$ (major). The absolute configuration was derived from comparison with literature data. ${ }^{15}$

$[\alpha]_{D}^{23}=+13\left(c=0.5\right.$ in $\mathrm{CHCl}_{3}, 90 \%$ ee $) ; \mathrm{R}_{\mathrm{f}}=0.49\left(5 \% \mathrm{MeOH}\right.$ in $\left.\mathrm{CH}_{2} \mathrm{Cl}_{2}\right) ; \mathrm{IR}$ (neat) $v_{\max } 3582,3377,3030,2970,2934,2879,1604$, 1496, 1453, 1384, 1092, 1063, 1047, 1031, 850, 762 and $701 \mathrm{~cm}^{-1}$; ${ }^{1} \mathrm{H}$ NMR $\delta: 1.37$ (d, J = $\left.7.1 \mathrm{~Hz}, 3 \mathrm{H}, \mathrm{Me}\right), 1.89$ (br s, $\left.1 \mathrm{H}, \mathrm{OH}\right)$, 3.10 (dquint, $J=20.0$ and $7.1 \mathrm{~Hz}, 1 \mathrm{H}, \mathrm{CHMe}), 3.61-3.82\left(\mathrm{~m}, 2 \mathrm{H}, \mathrm{CH}_{2} \mathrm{O}\right), 4.68(m, 1 \mathrm{H}, \mathrm{CHF}), 7.23-7.38(m, 5 \mathrm{H}, \mathrm{Ar}) ;{ }^{13} \mathrm{C} \mathrm{NMR} \delta$ : $17.4(\mathrm{~d}, J=6.3 \mathrm{~Hz}), 40.9(\mathrm{~d}, J=19.6 \mathrm{~Hz}), 63.4(\mathrm{~d}, J=22.7 \mathrm{~Hz}), 97.4(\mathrm{~d}, J=171.8 \mathrm{~Hz}), 126.8,127.9,128.4,141.8(\mathrm{~d}, J=2.1 \mathrm{~Hz}) ;{ }^{19} \mathrm{~F}$ NMR 8: -60.4 (m); MS (EI) (m/z) (rel. intensity) $168\left(\mathrm{M}^{+}+1,5 \%\right) 131(100 \%), 117(50 \%) 105(36 \%)$.

\section{Synthesis of complex VI:}

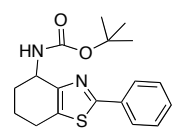

tert-Butyl 2-phenyl-4,5,6,7-tetrahydrobenzo[d] thiazol-4-ylcarbamate (6) A suspension of amide 5 (1.61 g, $6.23 \mathrm{mmol}, 1 \mathrm{eq})$, DMF $(16 \mathrm{~mL})$ and $t$ - $\mathrm{BuOH}(13 \mathrm{~mL})$ was heated in an oil bath at $70{ }^{\circ} \mathrm{C} . \mathrm{Pb}(\mathrm{OAc})_{4}(2.80 \mathrm{~g}, 6.32 \mathrm{mmol}, 1.01 \mathrm{eq})$ was added while stirring vigorously, and this was followed by slow dropwise addition of $\mathrm{Et}_{3} \mathrm{~N}(2.1 \mathrm{~mL})$. After stirring at $70{ }^{\circ} \mathrm{C}$ for 42 hours, $t-\mathrm{BuOH}$ and $\mathrm{Et}_{3} \mathrm{~N}$ were evaporated at reduced pressure, water $(40 \mathrm{~mL})$ together with acetic acid $(3 \mathrm{~mL})$ were added and after cooling in ice-bath, the solids were filtered off and washed with water $(30 \mathrm{~mL})$. Drying and chromatography $\left(\mathrm{CH}_{2} \mathrm{Cl}_{2} / \mathrm{MeOH}\right.$ 100:0 to 95:5) afforded 1.10 $\mathrm{g}$ (54\%) of 6 as white powder. 
$\mathrm{R}_{f}=0.58$ (pentane:EtOAc 75:25); IR (neat) $v_{\max } 3305,2983,2940,2868,2841,1689,1667,1464,1453,1365,1335,1245,1156$, 1064, 972, 874, 765 and $692 \mathrm{~cm}^{-1}$; ${ }^{1} \mathrm{H}$ NMR (400 MHz) $\delta: 1.49\left(\mathrm{~s}, 9 \mathrm{H},{ }^{\mathrm{t}} \mathrm{Bu}\right), 1.82-1.99\left(m, 3 \mathrm{H}, \mathrm{CH}_{2}\right), 2.18\left(m, 1 \mathrm{H}, \mathrm{CH}_{2}\right), 2.70-2.90$ $\left(m, 2 \mathrm{H}, \mathrm{CH}_{2}\right), 4.81-4.87$ ( $\left.m, 1 \mathrm{H}, \mathrm{CHN}\right), 5.05$ (br s, $\left.1 \mathrm{H}, \mathrm{NH}\right), 7.35-7.45(m, 3 \mathrm{H}, \mathrm{o} / \mathrm{p}-\mathrm{CH}), 7.85-7.91(m, 2 \mathrm{H}, \mathrm{m}-\mathrm{CH}) ;{ }^{13} \mathrm{C} \mathrm{NMR}(100$

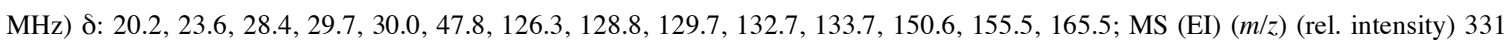
$\left(\mathrm{M}^{+}+1,100 \%\right), 274(69 \%), 229(68 \%), 212$ (34\%); Calculated for $\mathrm{C}_{18} \mathrm{H}_{22} \mathrm{~N}_{2} \mathrm{O}_{2} \mathrm{~S}$ C: 65.42, H: 6.71, N: 8.48; Found C: 65.12, H: $6.71, \mathrm{~N}: 8.33$.

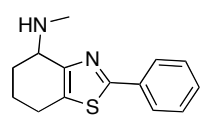

(R)-N-methyl-2-phenyl-4,5,6,7-tetrahydrobenzo[d]thiazol-4-amine (7). To a suspension of $\mathrm{LiAlH}_{4}(0.23 \mathrm{~g}, 6.0 \mathrm{mmol}, 4.0 \mathrm{eq})$ in THF $(10 \mathrm{~mL})$ was added the carbamate $6(0.50 \mathrm{~g}, 1.5 \mathrm{mmol}, 1.0 \mathrm{eq})$ in THF $(7 \mathrm{~mL})$ and the mixture was refluxed for $1.5 \mathrm{~h}$ until all starting material disappeared (followed by NMR). The reaction was cooled to RT, quenched with water ( $0.2 \mathrm{~mL}), 2 \mathrm{M} \mathrm{NaOH}$ (aq, 0.2 $\mathrm{mL})$ and water $(1 \mathrm{~mL})$. Suspension was filtered through celite and solids were washed by THF. Obtained solvents were evaporated, brine $(5 \mathrm{~mL})$ was added and the product was extracted with ether $(3 \times 20 \mathrm{~mL})$. After evaporation of solvent and chromatography $\left(\mathrm{CH}_{2} \mathrm{Cl}_{2} / \mathrm{MeOH} / \mathrm{Et}_{3} \mathrm{~N}\right.$ 98.5/1/0.5), $0.29 \mathrm{~g}$ (79\%) of 7 was obtained as colorless oil. The enantiomers were separated on preparative chiral HPLC; analytical column: Chiralcel OD-H, hexane: $i-\mathrm{PrOH} 90: 10,0.5 \mathrm{~mL} / \mathrm{min}, \mathrm{t}_{\mathrm{R}}=12.6 \mathrm{~min}$ and $17.5 \mathrm{~min}$; preparative column: Chiralcel OD, hexane: $i$-PrOH 85:15, $6 \mathrm{~mL} / \mathrm{min}, \mathrm{t}_{\mathrm{R}}=15.3 \mathrm{~min}$ and $21.7 \mathrm{~min}$. First enantiomer $(R):[\alpha]_{D}^{25}=-72\left(c=1.0\right.$ in $\left.\mathrm{CHCl}_{3}\right)$; second enantiomer $(S)[\alpha]_{D}^{26}=+69\left(c=1.0\right.$ in $\left.\mathrm{CHCl}_{3}\right) ; \mathrm{R}_{f}=0.50\left(\mathrm{CH}_{2} \mathrm{Cl}_{2} / \mathrm{MeOH} / \mathrm{Et}_{3} \mathrm{~N}\right.$ 97:2.5:0.5); IR (neat) $v_{\max } 3333,2943,1686$,

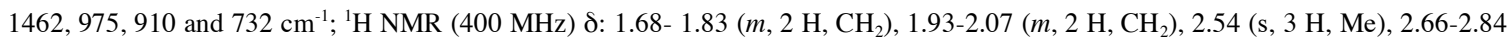
$\left(m, 2 \mathrm{H}, \mathrm{CH}_{2}\right), 3.13$ (br s, $\left.1 \mathrm{H}, \mathrm{NH}\right), 3.79(m, 1 \mathrm{H}, \mathrm{CHN}), 7.28-7.42(m, 3 \mathrm{H}, \mathrm{o} / \mathrm{p}-\mathrm{CH}), 7.80-7.89(m, 2 \mathrm{H}, \mathrm{m}-\mathrm{CH}) ;{ }^{13} \mathrm{C} \mathrm{NMR}(100$

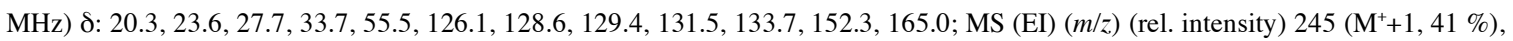
$215(100 \%), 201(8 \%)$

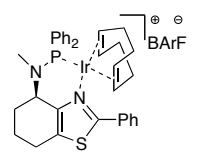

Thiazole Ir-complex (VI). Amine (R)-7 $(98 \mathrm{mg}, 0.40 \mathrm{mmol}, 1 \mathrm{eq})$ was co-evaporated with toluene $(2 \times 5 \mathrm{~mL})$ and redissolved in dry toluene $(4 \mathrm{~mL})$. Diisopropyl ethyl amine $(0.21 \mathrm{~mL}, 1.2 \mathrm{mmol}, 3 \mathrm{eq})$ was added and after immersing the mixture in ice bath, $\mathrm{Ph}{ }_{2} \mathrm{PCl}$ $(0.11 \mathrm{~mL}, 0.61 \mathrm{mmol}, 1.5 \mathrm{eq})$ was added. Reaction was stirred at $0{ }^{\circ} \mathrm{C}$ overnight, quenched with saturated $\mathrm{NaHCO}_{3}$ solution $(7 \mathrm{~mL})$, extracted with $\mathrm{CH}_{2} \mathrm{Cl}_{2}(3 \times 20 \mathrm{~mL})$ and dried over $\mathrm{Na}_{2} \mathrm{SO}_{4}$. Evaporation of solvent and chromatography (pentane $/ \mathrm{CH}_{2} \mathrm{Cl}_{2} / \mathrm{Et}_{3} \mathrm{~N}$ 90/10/0.5) gave $86 \mathrm{mg}$ of $\mathbf{8}$ as a colorless oil. After co-evaporation of $\mathbf{8}(86 \mathrm{mg}, 0.20 \mathrm{mmol}, 1.0 \mathrm{eq})$ with toluene $(2 \times 5 \mathrm{~mL})$, dry $\mathrm{CH}_{2} \mathrm{Cl}_{2}(8 \mathrm{~mL})$ and $[\mathrm{Ir}(\mathrm{COD}) \mathrm{Cl}]_{2}(67 \mathrm{mg}, 0.10,0.5 \mathrm{eq})$ were added and solution was refluxed for 1 hour under $\mathrm{N}_{2}$. After cooling to RT, water $(8 \mathrm{~mL})$ and $\mathrm{NaBAr}_{\mathrm{F}} * 3 \mathrm{H}_{2} \mathrm{O}(282 \mathrm{mg}, 0.3 \mathrm{mmol}, 1.5 \mathrm{eq})$ were added and the two-phase mixture was stirred vigorously for 30 minutes. The complex was extracted with $\mathrm{CH}_{2} \mathrm{Cl}_{2}(4 \times 10 \mathrm{~mL})$, dried $\left(\mathrm{Na}_{2} \mathrm{SO}_{4}\right)$ and purified by column chromatography on silica gel (pentane/ $\mathrm{CH}_{2} \mathrm{Cl}_{2} 50: 50$ to $30: 70$ ) to give $0.16 \mathrm{~g}(51 \%)$ of complex $\mathbf{V I}$ as bright orange crystals. $\mathrm{R}_{f}=0.38$ (pentane/ $\mathrm{CH}_{2} \mathrm{Cl}_{2} 50: 50$ ); $[\alpha]_{D}^{24}=-78.0\left(\mathrm{c}=1.0, \mathrm{CHCl}_{3}\right) ; \mathrm{IR}\left(\mathrm{CHCl}_{3}\right) v_{\max } 3436,2959,2928,1610,1455,1437,1355,1278,1218,1127,888$ and $764 \mathrm{~cm}^{-1} ;{ }^{1} \mathrm{H}$ $\operatorname{NMR}(400 \mathrm{MHz}) \delta:$ 1.25-1.42 (m, $\left.3 \mathrm{H}, \mathrm{CH}_{2}\right), 1.86-2.00\left(m, 2 \mathrm{H}, \mathrm{CH}_{2}\right), 2.05-2.18\left(m, 2 \mathrm{H}, \mathrm{CH}_{2}\right), 2.18-2.36\left(m, 4 \mathrm{H}, \mathrm{CH}_{2}\right), 2.51\left(\mathrm{~d}, J_{P}=\right.$ $7.6 \mathrm{~Hz}, 3 \mathrm{H}, \mathrm{Me}), 2.57(m, 1 \mathrm{H}), 2.69(\mathrm{ddd}, J=16.7,10.8$ and $5.7 \mathrm{~Hz}, 1 \mathrm{H}), 2.99(m, 1 \mathrm{H}), 3.08(m, 1 \mathrm{H}), 3.21(m, 1 \mathrm{H}), 4.08(m, 1 \mathrm{H}$, 
COD-CH), 4.66 ( $m, 1 \mathrm{H}, \mathrm{COD}-\mathrm{CH}), 5.69$ ( $m, 1 \mathrm{H}, \mathrm{COD}-\mathrm{CH}), 7.22-7.31(m, 3 \mathrm{H}, \mathrm{Ar})$, 7.34-7.47 ( $m, 5 \mathrm{H}, \mathrm{Ar})$, 7.49-7.60 ( $m, 11 \mathrm{H}, \mathrm{Ar})$,

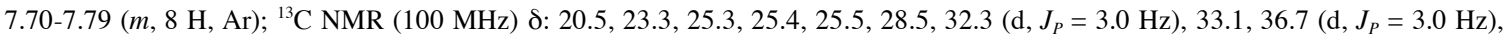
$55.7\left(\mathrm{~d}, J_{P}=14.5 \mathrm{~Hz}\right), 65.2\left(\mathrm{~d}, J_{P}=113.0 \mathrm{~Hz}\right), 89.7\left(\mathrm{~d}, J_{P}=13.7 \mathrm{~Hz}\right), 96.9\left(\mathrm{~d}, J_{P}=10.7 \mathrm{~Hz}\right), 117.4,120.5,123.2,125.1\left(\mathrm{~d}, J_{P}=61.0\right.$ $\mathrm{Hz}), 125.9,128.4,128.6,128.7,129.0,129.1,129.2,129.4,129.5,130.5,130.6\left(\mathrm{~d}, J_{P}=12.2 \mathrm{~Hz}\right), 131.3\left(\mathrm{~d}, J_{P}=10.8 \mathrm{~Hz}\right), 131.4$, 131.9, 132.0, 132.5, 132.9, 134.8, $151.2\left(\mathrm{~d}, J_{P}=1.5 \mathrm{~Hz}\right), 161.7\left(\mathrm{dd}, J_{F}=1.0\right.$ and $\left.0.5 \mathrm{~Hz}\right), 169.3$; Calculated for $\mathrm{C}_{66} \mathrm{H}_{49} \mathrm{BF}_{24} \mathrm{IrN}_{2} \mathrm{PS} \cdot 3 \mathrm{H}_{2} \mathrm{O}$ C: 48.15, H: 3.37, N: 1.70; Found C: 48.12, H: 3.67, N: 1.62.

\section{References}

(1) Inagaki, H.; Hakeda T; Miyauchi, R.; Kawakami, K.; Takahashi, H.; Takemura M. Heterocycles, 2004, 63, 699-706.

(2) Mong, S. M.; Leslie, A. K.; Vahak, H.; Boris, A. K; Charles, E. M. J. Fluorine Chem. 2005, 126, 1467-1475.

(3) Shigeki, S.; Katsuyuki, S.; Yoshimitsu, N. Tetrahedron Lett. 2003, 44, 3987-3990.

(4) Yanchang, S.; Yoefen, Z. J. Fluorine Chem. 1993, 61, 247-251

(5) Hanamoto T; Nishiyama K; Tateishi H; Kondo M. Synlett, 2001, 8, 1320-1322.

(6) Müller, C.; Stier, F; Weyerstahl P. Chem.Ber. 1977, 110, 124-137.

(7) Molchanov, A. P.; Kostilov, R. R. Zh. Org. Khim. 1993, 29, 510-517.

(8) Usuki, Y.; Fukuda, Y.; Iio, H. ITE Letters on Batteries, New Technologies \& Medicine, 2001, 2, 237-240.

(9) Sano, S; Ando T; Yokoyama K; Nagao Y. Synlett, 1998, 7, 777-779

(10) Normant, J. F.; Foulon, J. P.; Masure, D.; Sauvetre, R.; Villieras, J. Synthesis, 1975, 2, 122-5.

(11) Yoshimichi, N.; Tomoya, K.; Nobuo, I. J. Fluorine Chem. 1985, 29, 445- 458.

(12) Fujisawa, H.; Takeuchi, Y. J. Fluorine Chem. 2002, 117, 173-176.

(13) Beeson, T. D.; MacMillan, D. W. C. J. Am. Chem. Soc. 2005, 127, 8826-8828.

(14) Martinet, P.; Sauvetre, R.; Normant, J. F. J. Fluorine Chem. 1991, 52, 419432

(15) Huang, Y.; Walji, A. M.; Larsen, C. H.; MacMillan, D. W. C. J. Am. Chem. Soc. 2005, 127, 15051-15053. 